\title{
EVALUATION OF A MOBILE ELECTRODE FOR ELECTRICAL STIMULATION OF THE PERINEAL MUSCLE Preventive or Therapeutic Treatment
}

\author{
Fabiana S. B. Perez \\ School of Medicine, University of Brasilia, Brasilia, DF, Brazil \\ fabianasbp@hotmail.com \\ Adson F. da Rocha, Joao Luiz A. Carvalho, Patricia M. C. Paula \\ Department of Electrical Engineering, University of Brasilia, Brasilia, DF, Brazil \\ \{adson, joaoluiz, patricia.enge\}@gmail.com \\ Cleyciany B. Cruz \\ Faculty Padrão, Goiania, GO, Brazil \\ cleyci_sol@hotmail.com
}

Keywords: Perineal dysfunction, Electrical stimulation, Mobile electrode, Electrotherapy and physiotherapy.

\begin{abstract}
This work evaluates the effectiveness of a new type of electrode for functional electrical stimulation of the perineal muscle in women and in men. The new electrode is shaped like a pen, with an active stimulation electrode located on its tip. The goals of the study are to (i) demonstrate that stimulation using the new device results in increased muscle strength; and (ii) compare the performance of the new device with that of a traditional (fixed) electrode. Eight patients were evaluated, following a blind study protocol. The preliminary results suggest that stimulation with the new electrode achieves better results than stimulation with traditional electrodes, as higher increases in strength were observed in the group that used the mobile electrode for preventive treatment and as an option of therapeutic treatment for female and for male perineal dysfunction, in particular on erectile dysfunction in men with spinal cord injury.
\end{abstract}

\section{INTRODUCTION}

The perineum is a diamond-shaped muscle group located between the pubic symphysis, the ischial tuberosities, and the coccyx. It is separated from the pelvic cavity by a set of structures forming a pelvic diaphragm, which is composed by the pubococcygeus, iliococcygeus, and coccygeus muscles (Galhardo et al., 2007; Godec et al., 1975). These muscles occlude the pelvis, and provide support for the pelvic viscera when there is an increase in the intra-abdominal pressure due to physiological events such as coughing, bowel movements, laughing, carrying weight, pregnancy, birth delivery and sexual intercourse. This happens through the effective contraction and relaxation of the muscles of the pelvic floor. Perineal dysfunctions and psychosocial problems may occur if this coordinated action does not occur properly.
One solution to these dysfunctions is to strengthen the perineal muscles. This can be achieved through physical therapy techniques known as Arnold Kegel exercises (Moreno, 2004; Berghmans, 2006). However, some patients are unable to perform such exercises. In such cases, electrical stimulation may be used. Electrical stimulation of the perineal muscles has shown satisfactory therapeutic results in many etiology studies (Castro et al., 1998). Electrical stimulation has been widely used in the past years (Castro, 1998; Nielsen et al., 1992; Okada et al., 1992; Modotte et al., 1999) and has presented good results, especially using intracavitary electrodes. The performance of this technique depends on the choice of electrode, type of wave, amplitude, cycle, pulse, and frequency (Marques, 2008).

The treatment of perineal pathologies typically uses intracavitary electrodes. However, studies show 
adverse effects due to intra-cavity electrical stimulation with this type of electrode, such as: pain, unpleasant sensations, fecal incontinence, vaginal irritation and infection (Sand et al., 1995; Yamanish, 2000). Moreover, patients with spinal cord fall into a group of contraindication for electrical stimulation of the perineal intracavitary electrodes, Figure 1A, due to a sensory - motor change.

Due to these drawbacks, this work investigates an innovative non-intracavitary type of electrode: the mobile electrode, which uses a different mode of application.

This study compares a treatment that uses a fixed electrode to stimulate the muscles of the perineum with a treatment that uses a mobile pen-shaped electrode. The choice of electrodes is based on the study of Modotti et al. (1999). The mobile electrode has the size of a pen-tip, and can more adequately stimulate the motor point and the fibers of the small muscles in comparison with the fixed electrode, which stimulates only one or two muscles, and whose size makes its adaptation to the perineal region more difficult.

We evaluate the effectiveness of the fixed electrode and the pen-electrode in the recruitment of muscle fibers of the perineum and the time required to achieve a given level of strength. Preliminary results are presented. Improved muscle stimulation may help in curing and preventing diseases, by strengthening the muscle.

\section{METHODS}

The study was conducted at Cerei Clinical Center (Goiânia, GO, Brazil). The protocol was approved by the ethics and research committee of the Faculty of Medicine of the University of Brasilia. Eight volunteers of $37.5 \pm 7.65$ (mean \pm s.d.) years of age were chosen according to the evaluation of a questionnaire for inclusion or exclusion. The patients selected should be linked to the institution, between 25 and 50 years old, with no surgical correction of perineal pelvic floor disorders, sedentary, non-users of medical drugs other than contraception, sexually active, non-obese and have agreed to participate voluntarily in the research. The volunteers were blindly/randomly allocated into two groups, according to their arrival. After signing the consent, the volunteers were subjected to gynecological and strength evaluation using a Neurodyn Evolution perineal biofeedback device (Ibramed Ltda., Amparo, SP, Brazil) with a vaginal pressure probe (Ibramed Ltda., Amparo, SP,
Brazil). The probe was coated with a condom, and lubricated externally with carbogel. The device measures the maximum and minimum cavity pressure achieved by voluntary contraction of the perineum. After evaluation, the patient was subjected to the protocol of group A or B according to the assigned group.

The experimental protocol consists of two procedures, each applied to one of the two groups of subjects. In group A, the patients were subjected to electrical stimulation of the pelvic floor muscles by pen-electrode stimulation of five points around the vaginal opening in an arc that starts on one side of the groin and ends on the other side, passing through the centre of the pubic arch. This stimulates five motor points in different muscles, by moving the mobile pen-electrode during the thirty minutes of therapy. The five points of the perineal region are: the pubic region, central tendon, bilateral inguinal region, near the labium majus, and in the direction of the vaginal opening. The stimulator generates seventy impulses at each session toward a total of 14 pulses per point muscle during each session. The pen-electrode was fixed during six minutes at each point in each session.

In the use of mobile electrode for electrotherapeutic application in male patients with erectile dysfunction and partial Raqui-Medullar Trauma (RMT) is obtained satisfactory results, but with electrode application in the body of the penis, scrotum and central tendon.

Figure 1A shows the use of intracavitary electrode which in man should be anal with the patient positioned in lateral recumbence. Figure 1B shows the fixed external electrode positioned bilaterally in the groin which does not allow the visualization of local skin response. Figure 1C shows the mobile electrode in the penis body showing the swelling of the penis.

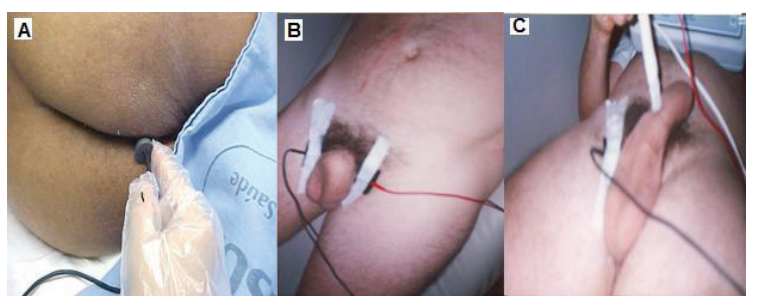

Figure 1: A) Intracavitary electrode; B) Fixed electrode; C) Mobile electrode.

The electrode intensity instead of going on the threshold of the patient pain, it goes to the moment when the therapist visualizes the scrotal contraction or the swelling of the penis, Figure $1 \mathrm{C}$. 
Group B participants were subjected to electrical stimulation using fixed electrodes. A group of four electrodes, arranged in a cross shape, were placed around the vaginal opening, thus stimulating only two muscles. The two electrodes from cable 1 were arranged on the central tendon and the pubis, thus stimulating this region. The two electrodes from cable 2 bilaterally stimulate the groin, closing the vaginal opening. Seventy impulses were used to simultaneously stimulate these four points during thirty minutes of therapy.

A Neurodyn Ruby stimulator (Ibramed Ltda., Amparo, SP, Brazil) was configured as follows for both groups: low frequency synchronous FES with the following envelope: frequency $=20 \mathrm{kHz}$; period $=50 \mu \mathrm{s} ;$ rise $=7 \mathrm{~s}$; on $=10 \mathrm{~s}$; decay $=10 \mathrm{~s}$; off $=5 \mathrm{~s}$; time $=30$ minutes. The current intensity was adjusted according to each patient's sensibility, provided that the electrical stimulation reaches the motor threshold of the muscle without triggering a painful stimulus. A vaginal probe was introduced in the vagina of the patient during electrotherapy, using a Neurodyn Evolution perineal biofeedback device (Ibramed Ltda., Amparo, SP, Brazil) to check the pressure before and after vaginal electrotherapy in order to observe whether there is increase in strength or muscle fatigue caused by the electrical stimulation. The FES frequency was chosen due to its extensive use in the literature (Godec et al., 1975; Nielsen et al., 1992; Okada et al., 1999, Yamanish et al. 2000). The protocol was repeated in 12 sessions, performed twice a week, at a total time of 30 minutes per session.

A comparative analysis of muscle fatigue of the subjects of group A compared to group B was performed. For each subject, the intra-session increase in muscle strength was calculated as the difference between end pressure (after electrical stimulation) and initial pressure (before electrical stimulation). The inter-session increase in muscle strength was evaluated by linear regression of the measured pressures from each subject.

\section{RESULTS AND DISCUSSIONS}

This work presents current results from an ongoing study. Therefore, only data from the first 7 experimental sessions (out of 12) are presented. However, the study is still in progress and complete results will be published at the end of the experiments.

Local muscle fatigue is a decrease in muscle response to a repeated stimulus, i.e. a normal physiological response of muscle is characterized by a decrease in amplitude of motor unit potential (Kisner, 2009). Table I presents and the angular coefficient of the linear regression on the 7 measurements (sessions) from each subject. Positive angular coefficients indicate a tendency of increasing pressure with each additional session, i.e., an increase in muscle strength due to periodic electrotherapy. Three out of four subjects in group A presented positive angular coefficients, while only one out four subjects in group B presented positive slopes.

Table 1: Initial pressure in each session (in $\mathrm{mmHg}$ ) and corresponding linear regression angular coefficients.

\begin{tabular}{|c|c|c|c|c|c|c|c|c|c|}
\hline \multirow{2}{*}{ Group } & Session & S1 & S2 & S3 & S4 & S5 & S6 & S7 & $\begin{array}{c}\text { Angular } \\
\text { coefficient }\end{array}$ \\
\hline & Subject 1 & 13 & 113 & 47 & 57 & 132 & 69 & 93 & -4.6071 \\
\hline \multirow{2}{*}{ A } & Subject 2 & 64 & 39 & 92 & 77 & 73 & 72 & 84 & 3.8214 \\
\hline & Subject 3 & 41 & 49 & 41 & 42 & 45 & 42 & 64 & 3.8214 \\
\hline & Subject 4 & 51 & 50 & 62 & 39 & 48 & 51 & 56 & 0.1071 \\
\hline \multirow{2}{*}{ B } & Subject 1 & 38 & 46 & 40 & 40 & 30 & 35 & 31 & -1.8929 \\
\hline & Subject 2 & 1 & 40 & 52 & 2 & 14 & 72 & 87 & 10.1429 \\
\hline & Subject 3 & 79 & 76 & 131 & 106 & 101 & 105 & 2 & -7.2500 \\
\hline & Subject 4 & 56 & 62 & 63 & 49 & 46 & 62 & 55 & -0.7143 \\
\hline
\end{tabular}

The measured pressure at the beginning of each session for each subject of group $\mathrm{A}$ is showed in Figure 2 and for group B in Figure 3. These results suggests a trend of increased strength between successive sessions for subjects stimulated with the mobile electrode (group A), and a trend of strength loss in subjects stimulated with the fixed electrode (group B). The mobile electrode was expected to provide higher strength gain, because force production is directly related to the number of stimulated motor points [8].

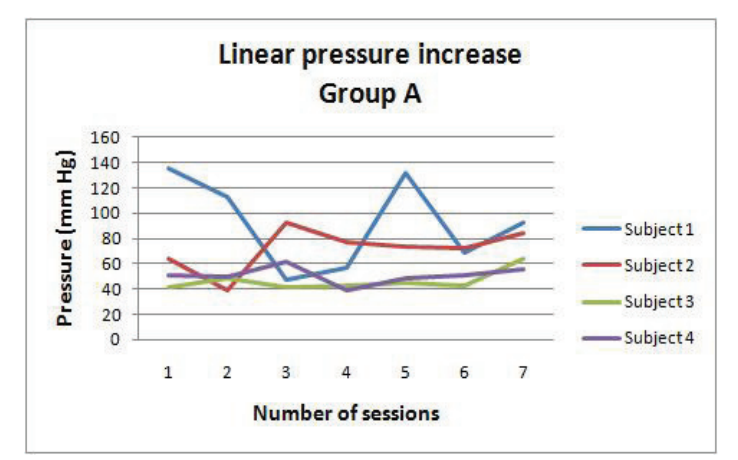

Figure 2: Measured muscle pressure at the beginning of each session for each subject of group A. 


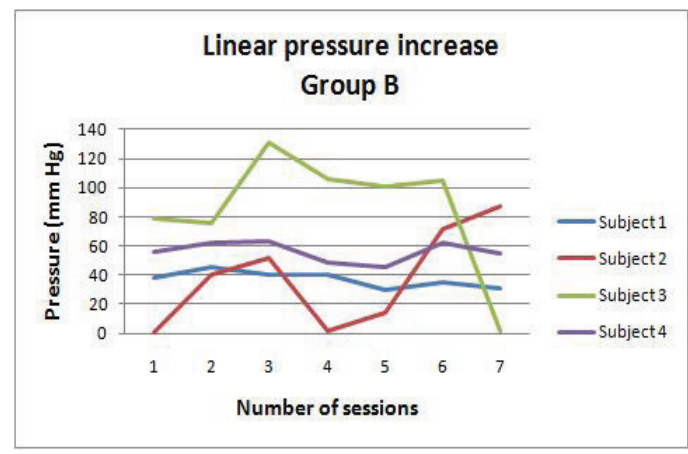

Figure 3: Measured muscle pressure at the beginning of each session for each subject of group B.

Treatment with intracavitary electrode is not indicated for patients with altered sensitivity because it might cause injury which will not be perceived by the therapist and/or by the patient, on the other hand with the conventional electrode (fixed) it might cause injure due to the static positioning on the skin surface during the therapy without the visualization of the skin surface of the patient with altered sensitivity, Figure 1B.

With the new mobile electrode (pen), the electrical load is greater at the tip of the conductor with an intense electric field which can trigger faster the action potential in the injured muscle, and because of being mobile and external even with changing sensory of the patient, the therapist is able to visualize the muscle contraction even though the patient does not notice, and monitor the dermal reaction of it to prevent injury, Figure 1B.

\section{CONCLUSIONS}

Preliminary results suggest that the pen-electrode is more effective than the fixed electrode in providing gain of perineum muscle strength. The comparison was performed using similar protocols with respect to duration and current administration, differing only for the fact that, with the pen-electrode, each point received 14 stimulated impulses during the thirtyminute pulse therapy, while seventy impulses were used for each of the four points during the sessions with the fixed electrode.

The preliminary results suggest that the penelectrode may be a better option for electrotherapy than the fixed electrode in the prevention and in the treatment of perineal dysfunction of, highlighting the possibility of the use in patients with reduced sensitivity, for example, the spinal cord injured.

The group that received stimulation with fixed electrodes showed a reduction in strength, suggesting a slower perineal muscle response due to muscle fatigue, which occurs during prolonged simultaneous stimulation of the four fixed points. The mobile pen-electrode stimulates five points nonsimultaneously. The increased strength resulting from a minor muscle fatigue can possibly shorten the therapy duration and reduce the financial costs to the patient.

\section{REFERENCES}

Berghmans B. The role of the pelvic physical therapist. Acta Urol Esp, 2006. 30:110-22.

Castro,R. A. Arruda,R. M.,Takano,C.C.,Girão,M. J. B. C.,Sartori,M. G.,Baracat,E. C.,Lima,G. R. Tratamento da incontinência urinária com eletroestimulação. In: Gineco.Obstet.Atual. v.7. n.4, abr.1998. p. 49-50.

Galhardo,C.,Katayama,M. Anatomia e fisiologia do trato urinário inferior feminino. In:Chiarapa,T. R.,Cacho, D. P.,Alves,A. F. D. Incontinência urinária Feminina: Assistência Fisioterapêutica e Multidiciplinar. São Paulo: Livraria Médica Paulista Editora, 2007.

Godec C, Cass A. S, Ayala G. F. Bladder inhibition with functional electrical stimulation. Urolog, 1975. 6:663-6.

Kisner, Carolyn, Fundamentos da exercícios terapêuticos e técnicas, $5^{\circ}$ edição, Editora Manole, 2009.

Marques, A. A. A estimulação do nervo tibial posterior no tratamento da bexiga hiperativa. Unicamp. Campinas, SP: [s.n.], 2008.

Modotte,W. P.,Moreira,E. C. H.,Pascon,A. M.,Dias,R.,Pascotini,C.,Filho,C. I. S.,Braga,M. A. Incontinência urinária - trabalho conservador. In: ginecol.Obstet.Atual. v.8. n.6, 1999. p. 6-13

Moreno, Adriana L. Fisioterapia em Uroginecologia, Barueri, SP, Manole, 2004.

Nielsen M, Samuelsson S. M. Maximal electrical stimulation of patients with frequency, urgency and urge incontinence. Acta Obstet Gynecol Scand, 1992. 71:629-31.

Okada N, Igawa Y, Nishizawa O. Functional electrical stimulation for detrusor instability. In: Urogynecol $J$, 1999. 10:329-35.

Sand P. K, Richardson D. A, Staskin D. R, Swift S. E, Appel RA, Whitmore KE et al. Pelvic floor electrical stimulation in treatment of genuine stress incontinence: a muticenter placebo-controlled trial. Am J Obstet Gynecol: 1995. 173:72-9.

Yamanish T, Yasuda K, Hattori T, Suda S. Randomized, double-blind study of electrical stimulation for urinary incontinence due to detrusor overactivity. Urology. 2000, 55:353-7. 